\title{
Searching for additional Higgs bosons via Higgs cascades
}

\author{
Christina Gao, Markus A. Luty, Michael Mulhearn, Nicolás A. Neill, and Zhangqier Wang \\ Physics Department, University of California, Davis, California 95616, USA
}

(Received 16 July 2017; published 27 April 2018)

\begin{abstract}
The discovery of a $125 \mathrm{GeV}$ Higgs boson at the Large Hadron Collider strongly motivates direct searches for additional Higgs bosons. In a type I two Higgs doublet model there is a large region of parameter space at $\tan \beta \gtrsim 5$ that is currently unconstrained experimentally. We show that the process $g g \rightarrow H \rightarrow A Z \rightarrow Z Z h$ can probe this region, and can be the discovery mode for an extended Higgs sector at the LHC. We analyze 9 promising decay modes for the $Z Z h$ state, and we find that the most sensitive final states are $\ell \ell \ell \ell b b, \ell \ell j j b b, \ell \ell \nu \nu \gamma \gamma$ and $\ell \ell \ell \ell+$ missing energy.
\end{abstract}

DOI: 10.1103/PhysRevD.97.075040

\section{INTRODUCTION}

The discovery of a Higgs-like $125 \mathrm{GeV}$ particle at the Large Hadron Collider (LHC) began a new era in elementary particle physics $[1,2]$. The experiments have moved rapidly from discovery to precision measurements of the properties of the new particle. We now have strong evidence that the $125 \mathrm{GeV}$ particle is a scalar, and we know that its couplings agree with the couplings of the Standard Model (SM) Higgs at the 15\% level [3,4]. This implies that electroweak symmetry breaking is dominantly a weakly-coupled phenomenon.

As a direct result of this, for the first time in the history of particle physics we have an experimentally complete theory that can be consistently extrapolated to energies far above the $\mathrm{TeV}$ scale. This is a tremendous achievement, but it also raises the question of whether there is any additional new physics to be found at the LHC. The existence of an elementary scalar particle brings to the fore the problem of naturalness of the standard model, and strongly motivates new physics at the weak scale accessible to the LHC. In particular, extensions of the standard model that address the naturalness problem generally require an extension of the Higgs sector (for example SUSY or PNGB Higgs models). If the weak scale instead has an anthropic explanation, we do not expect any additional light scalars. Searching for additional Higgs bosons is therefore an important part of the program of probing naturalness at the LHC.

There are many candidate models for physics beyond the standard model, but the absence of any signal has ruled out the simplest and most natural models. This motivates

Published by the American Physical Society under the terms of the Creative Commons Attribution 4.0 International license. Further distribution of this work must maintain attribution to the author(s) and the published article's title, journal citation, and DOI. Funded by SCOAP ${ }^{3}$. searching as widely as possible for new physics, and suggests a phenomenological approach of looking for signals based on simple extensions of the standard model. It is important not to miss any possible signal, and at a hadron collider like the LHC one has to know exactly what to look for to find new physics. Simplified models that contain the minimum degrees of freedom relevant for a particular type of signal are useful tools for generating signal events and interpreting the results of searches [5]. This is the philosophy adopted in the present paper.

Our simplified model for additional Higgs bosons is the two Higgs doublet model (2HDM). This is the simplest model that has an additional source of electroweak symmetry breaking beyond the standard model. In this paper, we study the Higgs cascade process $g g \rightarrow$ $H \rightarrow Z A \rightarrow Z Z h$. We demonstrate that this can be important in a type I $2 \mathrm{HDM}$ for $\tan \beta \gtrsim 5$. Of course, the masses must be such that the decay is allowed, and we must have $m_{A}<2 m_{t}$, otherwise the decay $A \rightarrow t \bar{t}$ dominates and reduces our signal. Our search is therefore sensitive in the mass region

$$
215 \mathrm{GeV} \lesssim m_{A} \lesssim 355 \mathrm{GeV}, \quad m_{H} \gtrsim m_{A}+90 \mathrm{GeV}
$$

This is a region of parameter space where other direct searches are insensitive. We demonstrate that the searches we consider can be more sensitive than indirect constraints from Higgs coupling measurements, and in fact can be the discovery mode for new Higgs bosons. We analyzed 9 different final states for the $Z Z h$ decays that we believe are the most promising. These are listed in Table I. We use the notation $Z Z h \rightarrow\left(\ell^{+} \ell^{-}\right)(\nu \nu)(b \bar{b})$ to indicate $Z \rightarrow \ell^{+} \ell^{-}$, $Z \rightarrow \nu \nu, h \rightarrow b \bar{b}$, etc., where $\ell=e, \mu$. We find that the most sensitive final state is the "golden mode" $\ell^{+} \ell^{-} \ell^{+} \ell^{-} b \bar{b}$, which is essentially background-free, and 
TABLE I. Final states of $Z Z h$ considered in this paper, with comments and signal significance for $14 \mathrm{TeV}$ LHC with integrated luminosity of $300 \mathrm{fb}^{-1}$.

\begin{tabular}{llc}
\hline \hline$Z Z h$ decay modes & \multicolumn{1}{c}{ Comments } & Significance \\
\hline$\left(\ell^{+} \ell^{-}\right)\left(\ell^{+} \ell^{-}\right)(b \bar{b})$ & clean, ideal for reconstruction & $11 \sigma$ \\
$\left(\ell^{+} \ell^{-}\right)(j j)(b \bar{b})$ & large signal and background & $3.6 \sigma$ \\
$\left(\ell^{+} \ell^{-}\right)(\nu \nu)(b \bar{b})$ & overwhelmed by $t \bar{t}$ background & small \\
$\left(\ell^{+} \ell^{-}\right)(j j)\left(\tau_{h} \tau_{h}\right)$ & overwhelmed by jet-faked $\tau$ background & $0.7 \sigma$ \\
$\left(\ell^{+} \ell^{-}\right)(\nu \nu)\left(\tau_{h} \tau_{h}\right)$ & not enough signal yield & small \\
$\left(\ell^{+} \ell^{-}\right)(j j)(\gamma \gamma)$ & relatively clean, small signal cross section & $1.7 \sigma$ \\
$(\nu \nu)(j j)(\gamma \gamma)$ & hard to reconstruct & $0.5 \sigma$ \\
$\left(\ell^{+} \ell^{-}\right)(\nu \nu)(\gamma \gamma)$ & clean but very small cross section \\
$\ell^{+} \ell^{-} \ell^{+} \ell^{-}+E_{\mathrm{T}}$ & relatively clean after hard $\mathscr{E}_{\mathrm{T}}$ cut & $2.8 \sigma$ \\
\hline \hline
\end{tabular}

can lead to a $5 \sigma$ discovery of our our benchmark model at $66 \mathrm{fb}^{-1}$ at the $14 \mathrm{TeV}$ LHC. Perhaps more surprisingly, we also find that the final state $\ell^{+} \ell^{-} j j b \bar{b}$ is also sensitive. This mode has large background, and a careful treatment of background uncertainty is needed to draw any conclusion. We obtain a $3 \sigma$ sensitivity with $300 \mathrm{fb}^{-1}$ with a cut-andcount analysis, but we show that a boosted decision tree technique may be able to boost the significance above $5 \sigma$. We also find that the final states $\left(\ell^{+} \ell^{-}\right)(\nu \nu)(\gamma \gamma)$ and $\ell^{+} \ell^{-} \ell^{+} \ell^{-}+E_{\mathrm{T}}$ are sensitive at the $3 \sigma$ and $2 \sigma$ level, respectively. Searches for $Z Z h$ are therefore very strongly motivated in the current run of the $14 \mathrm{TeV}$ LHC.

Because several of the searches are rate-limited, the highluminosity LHC (3000 $\mathrm{fb}^{-1}$ at $\left.14 \mathrm{TeV}\right)$ is very effective in exploring the $Z Z h$ signal. We find that all five of the modes listed above can give a $5 \sigma$ signal for our benchmark model, illustrating the possibility of studying the signal in multiple channels.

This paper is organized as follows. In Sec. II, we briefly review the $2 \mathrm{HDM}$ and explain why the $Z Z h$ final state is a sensitive probe when in a type I $2 \mathrm{HDM}$ at large $\tan \beta$. In Sec. III, we report our analysis of experimental searches in a number of final states and compute the significance for the $14 \mathrm{TeV}$ LHC. Section IV contains our outlook and conclusions.

\section{ADDITIONAL HIGGS BOSONS IN THE TWO HIGGS DOUBLET MODEL}

In this section we briefly review the experimental constraints on the two Higgs doublet model (2HDM) and motivate our search. The 2HDM extends the SM by adding an additional Higgs doublet. It is by now a textbook topic [6].

There are many parameters in a general 2HDM, but only a small number of them are the most important for phenomenology. One important parameter of the model is the ratio of the vacuum expectation values (VEVs) of the two Higgs doublets $H_{1,2}$, conventionally parametrized by $\tan \beta=v_{2} / v_{1}$, with $v=\sqrt{v_{1}^{2}+v_{2}^{2}}=246 \mathrm{GeV}$. Another important parameter is the mixing angle $\alpha$ that defines the mass eigenstates of the neutral $C P$ even scalar states:

$$
\left(\begin{array}{c}
H^{0} \\
h^{0}
\end{array}\right)=\left(\begin{array}{cc}
\cos \alpha & \sin \alpha \\
-\sin \alpha & \cos \alpha
\end{array}\right)\left(\begin{array}{c}
H_{1}^{0} \\
H_{2}^{0}
\end{array}\right) .
$$

For the interactions of the Higgs bosons with gauge bosons and other Higgs bosons, there is no natural choice for $H_{1,2}$, so $\alpha$ and $\beta$ are not separately meaningful. What is meaningful is the mixing angle between the mass basis $\left(h^{0}, H^{0}\right)$ and the basis where one of the fields has vanishing VEV. This "Higgs angle" is given by $\theta_{H}=\beta-\alpha+\pi / 2$. (The $\pi / 2$ is due to unfortunate standard conventions.) The coupling of all of the Higgs bosons to vector bosons is therefore determined by $\beta-\alpha$. Some examples relevant for our work are

$$
\begin{array}{ll}
A h Z \propto \cos (\beta-\alpha), & A H Z \propto \sin (\beta-\alpha), \\
h Z Z \propto \sin (\beta-\alpha), & H Z Z \propto \cos (\beta-\alpha) .
\end{array}
$$

The fact that the $h Z Z$ coupling is modified for $\cos (\beta-\alpha) \neq 0$ is an important constraint on this model. In the "alignment limit" $\cos (\beta-\alpha) \rightarrow 0$ the light mass eigenstate $h^{0}$ is solely responsible for electroweak symmetry breaking, and so its couplings to all states are standard-model like.

The parameters $\alpha$ and $\beta$ become separately meaningful when we consider Yukawa couplings between the Higgs fields and fermions, which pick out particular linear combinations of $H_{1,2}$. The most well-studied possibilities are so-called type I models where $\mathrm{H}_{2}$ couples to all fermions, and type II models where $H_{1}$ couples to leptons and down-type quarks, and $H_{2}$ couples to up-type quarks. Type II models have received the most attention because the minimal supersymmetric standard model (MSSM) Higgs sector is type II, but extensions of the MSSM can have have an effective type I Higgs sector at low energies [7-9]. There are other possibilities beyond type I and II, but these are sufficient to illustrate the physics being studied here.

In type I models, the coupling of neutral Higgs fields to fermions are 


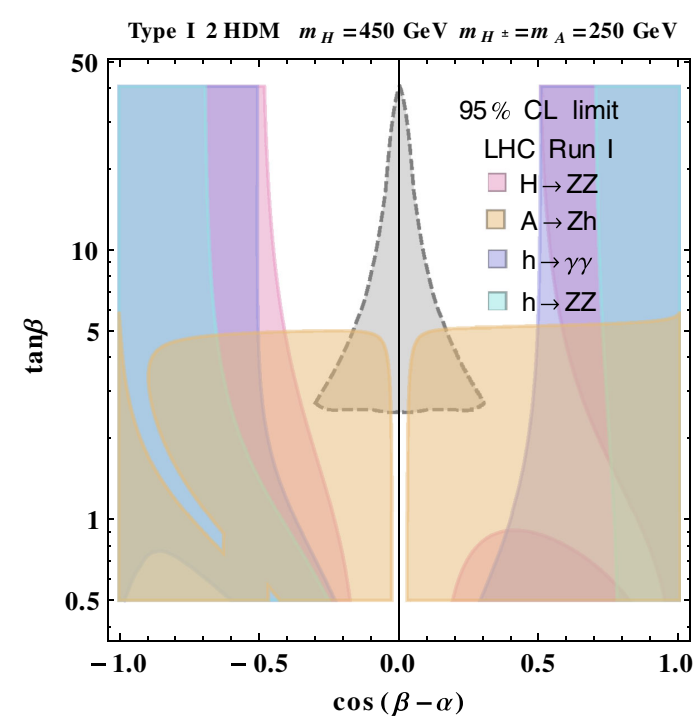

(a)

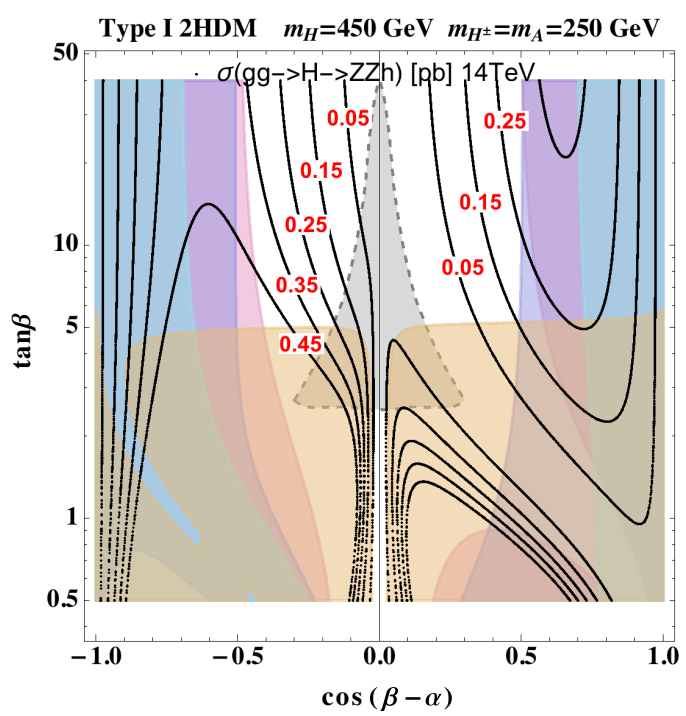

(b)

FIG. 1. Status of type I 2HDM after the $8 \mathrm{TeV}$ run of the LHC for $m_{A}=m_{H^{ \pm}}=250 \mathrm{GeV}, m_{H}=450 \mathrm{GeV}$. Plotted on the right are the values of the $\sigma \times \mathrm{BR}$ for $g g \rightarrow H \rightarrow A Z \rightarrow Z Z h$. The dashed grey region near $\cos (\beta-\alpha)=0$ shows a region where some of the Higgs couplings are becoming large and the theory is losing perturbativity (see Appendix A for details).

$f f h \propto \frac{\cos \alpha}{\sin \beta}, \quad f f H \propto \frac{\sin \alpha}{\sin \beta}, \quad f f A \propto \cot \beta$,

while in type II models the couplings depend on the type of fermion:

$$
\begin{aligned}
u u h \propto \frac{\cos \alpha}{\sin \beta}, & d d h, \ell \ell h \propto \frac{\sin \alpha}{\cos \beta}, \\
u u H \propto \frac{\sin \alpha}{\sin \beta}, & d d H, \ell \ell H \propto \frac{\cos \alpha}{\cos \beta}, \\
u и A \propto \cot \beta, & d d A, \ell^{+} \ell^{-} A \propto \tan \beta .
\end{aligned}
$$

The values of $\alpha$ and $\beta$ determine much of the phenomenology, and we will plot constraints in the plane of $\cos (\beta-\alpha)$ and $\tan \beta$. The vertical line $\cos (\beta-\alpha)=0$ is the alignment limit, which is very difficult to probe experimentally. In Figs. 1 and 2 we show the experimental constraints from the $8 \mathrm{TeV}$ run of the LHC on type I and II 2HDM models, for the benchmark masses $m_{H}=450 \mathrm{GeV}$ and $m_{A}=m_{H^{ \pm}}=250 \mathrm{GeV}$. The indirect constraints from $h \rightarrow \gamma \gamma$ and $h \rightarrow Z Z$ are independent of the masses of the heavy Higgs bosons, while the direct search constraints from $H \rightarrow Z Z$ and $A \rightarrow Z h$ weaken rapidly when this mass is increased. Both direct and indirect probes are therefore essential for probing the Higgs sector.

In type I models, we see from Fig. 1 that there is a large region of parameter space of the model at large $\tan \beta$ that is consistent with all theoretical constraints, but currently unconstrained experimentally. The indirect constraints from $h \rightarrow \gamma \gamma$ and $h \rightarrow Z Z$ are approximately independent of $\tan \beta$ in this region. The constraint from $A \rightarrow Z h$ becomes ineffective at large $\tan \beta$ because the Att coupling becomes small, suppressing the dominant production gluon fusion production of $A$. Constraints from $h \rightarrow \tau \tau$ and $h \rightarrow$ $b b$ are also not important because their branching ratio is not strongly affected at large $\tan \beta$, unlike in type II models. The production of $H$ is unsuppressed, and the process $H \rightarrow$ $Z Z$ is the dominant direct constraint, but its effectiveness is limited by the large background from SM ZZ production.

Figure 2 shows the same constraints for a type II model. The indirect constraints are much more constraining in this case, and there is much less parameter space open for direct searches. This is mainly due to the fact that for large $\tan \beta$ the $h b \bar{b}$ coupling becomes large there, which affects the $h$ branching ratios.

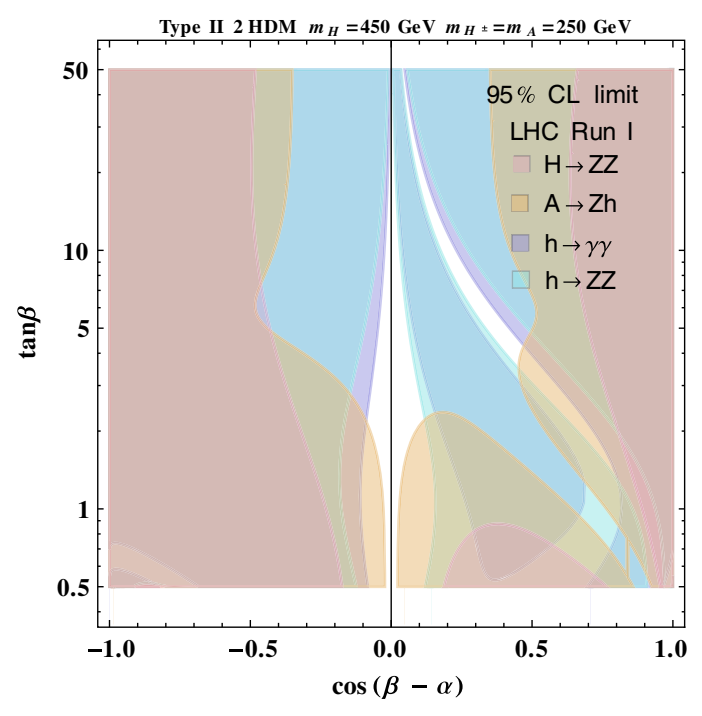

FIG. 2. Status of type II $2 \mathrm{HDM}$ after the $8 \mathrm{TeV}$ run of the LHC for $m_{A}=m_{H^{ \pm}}=250 \mathrm{GeV}, m_{H}=450 \mathrm{GeV}$. 
In this paper we investigate the cascade decay $H \rightarrow$ $Z A \rightarrow Z Z h$ as a way of probing the unconstrained region of the type I 2HDM. The motivation for this is that the $H$ production is unsuppressed at large $\tan \beta$. The decay $A \rightarrow$ $Z h$ is suppressed for $\cos (\beta-\alpha) \rightarrow 0$, but is still significant for much of the unexplored region. This can be seen in Fig. 1(b), which shows the $\sigma \times \mathrm{BR}$ for $g g \rightarrow H \rightarrow Z A \rightarrow$ $Z Z h$ superimposed on the other experimental constraints. We will demonstrate that this is more effective than $g g \rightarrow$ $H \rightarrow Z Z$ in this parameter range, because the latter suffers from larger standard model backgrounds.

To compare the various channels, we use the benchmark mass values $m_{A}=m_{H^{ \pm}}=250 \mathrm{GeV}, m_{H}=450 \mathrm{GeV}$. We choose the other parameters in the $2 \mathrm{HDM}$ potential so that the decay $H \rightarrow h h$ is negligible, which is easily accomplished without any large couplings or tunings. This benchmark model has a $\sigma \times \mathrm{BR}$ for $Z Z h$ production of $0.45 \mathrm{pb}$. We also give results from a scan over $m_{H}$ at the end of the paper.

In Appendix A we show that the only theoretical constraint on this benchmark model is that for large $\tan \beta$ near $\cos (\beta-\alpha)$ some of the Higgs quartic couplings are becoming large. (This region is shown in Fig. 1.) This is happening because one of the Higgs doublets is decoupling in this limit, while our benchmark is holding the electroweak-breaking mass splittings in this doublet fixed. In this region the model is not under full theoretical control, but we believe that it is still interesting to search in this region.

\section{SEARCHING FOR ZZh FINAL STATES}

To examine the experimental prospects for $Z Z h$ production, we calculate the expected signal significance for the benchmark model with $m_{A}=250 \mathrm{GeV}$ and $m_{H}=450 \mathrm{GeV}$, which has $\sigma \times \mathrm{BR}(Z Z h)=0.45 \mathrm{pb}$ for the $14 \mathrm{TeV}$ LHC, with total luminosity of $300 \mathrm{fb}^{-1}$, as reported in Table I. Simulated events for both signal and SM backgrounds were generated by MADGRAPH5 [10], with showering and hadronization simulated by PYTHIA8 [11], and the detector response simulated by DeLPHES3 [12]. For the multijet backgrounds, jet matching was used. The tagging rate, particle reconstruction efficiency and particle isolation requirement are reported by CMS in Run I [13-17]. The LO cross-sections of the SM backgrounds for each channel are calculated by MADGRAPH5.

To distinguish the Higgs Cascade signal over much larger SM background, we apply event selection requirements, separately optimized for each final state, as described below.

\section{A. Requirements on reconstructed particles}

Detector limitations, such as trigger requirements and detector acceptance, are common across all channels, and so common selection requirements are applied to reconstructed jets, muons, electrons, and taus, before further selection requirements, optimized for each final state, are applied.

Leptons are required to have $p_{\mathrm{T}}>10 \mathrm{GeV}$ and pseudorapidity $|\eta|<2.5$. We further require isolated leptons, as determined from the isolation ratio $R_{\mathrm{iso}}=p_{\mathrm{T}, j} / p_{\mathrm{T}, \ell}$ where $p_{\mathrm{T}, j}$ is the clustered transverse energy, contained in a cone of radius $\Delta R$ around the lepton, and $p_{\mathrm{T}, \ell}$ is the lepton transverse energy. The lepton isolation requirement used in this analysis is $\Delta R<0.3$ with $R_{\text {iso }}<0.1$, except for signals where both $Z$ 's decay to leptons. In this case, the leptons are boosted in the $Z$ direction and therefore tend to be close together. We therefore use a different isolation requirement with $\Delta R<0.2$, with $R_{\text {iso }}<0.05$ for electrons and $R_{\text {iso }}<0.1$ for muons. Similar isolation criteria have been used by ALTAS for their multilepton searches in LHC Run I $[18,19]$.

Jets are required to satisfy $p_{\mathrm{T}}>20 \mathrm{GeV}$ and $|\eta|<5$. Photons are required to satisfy $p_{\mathrm{T}}>10 \mathrm{GeV}$ and $|\eta|<2.5$. The $b$-tagging rate is adjusted to be the same as one of the commonly used $b$-taggings in CMS detector, which is $75 \%$ tagging efficiency and $1.5 \%$ misidentification rate [13]. According to the $Z \rightarrow \tau \tau$ cross section measurement in CMS run II, the hadronic decayed tau identification efficiency is set to be $60 \%$ and the misidentification rate from jets is set to be $3 \%$.

The remaining event selection is optimized for each individual channel, as described below.

$$
\text { B. } Z Z \boldsymbol{Z h} \rightarrow\left(\boldsymbol{\ell}^{+} \boldsymbol{\ell}^{-}\right)\left(\boldsymbol{\ell}^{+} \boldsymbol{\ell}^{-}\right)(\boldsymbol{b} \overline{\mathrm{b}})
$$

This is the "golden channel" for the $Z Z h$ final state, with a clean signal and essentially no background. For this channel, we require two $b$-tagged jets and two oppositesign same-flavor (OSSF) isolated lepton pairs. The main backgrounds for this channel come from $t \bar{t} Z$ and $Z Z+b \bar{b}$. As the probability of detecting a $b$ quark from misidentification of a jet is about $1.5 \%$, the $Z Z+$ jets with two misidentified $b$ jets is much smaller than $Z Z+b \bar{b}$. To ensure that the trigger efficiency is nearly $100 \%$ for the selected events, we require that the leading lepton has $p_{\mathrm{T}}>20 \mathrm{GeV}$, while the second has $p_{\mathrm{T}}>15 \mathrm{GeV}$.

The remaining requirements were chosen to maximize the significance, approximated by

$$
Z=\sqrt{2\left[(S+B) \times \ln \left(1+\frac{S}{B}\right)-S\right]}
$$

where $S$ is the number of signal events and $B$ is the number of background events, at $300 \mathrm{fb}^{-1}$. To suppress the $t \bar{t} Z$ background, the total missing transverse energy is required to satisfy $E_{\mathrm{T}}<80 \mathrm{GeV}$.

In this final state, all of the intermediate particles in the Higgs cascade are fully reconstructed. This channel is therefore nearly background free after cuts on the invariant mass of the reconstructed $H$ and $A$. The invariant mass of 


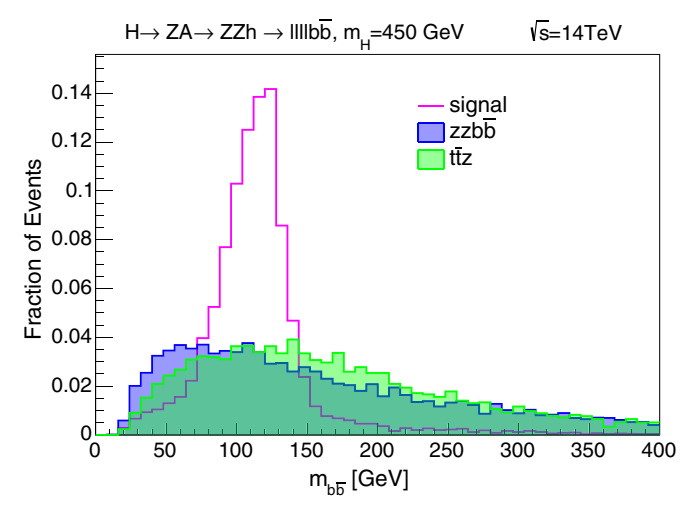

(a) Di-bottom invariant mass distributions. All normalized to 1.

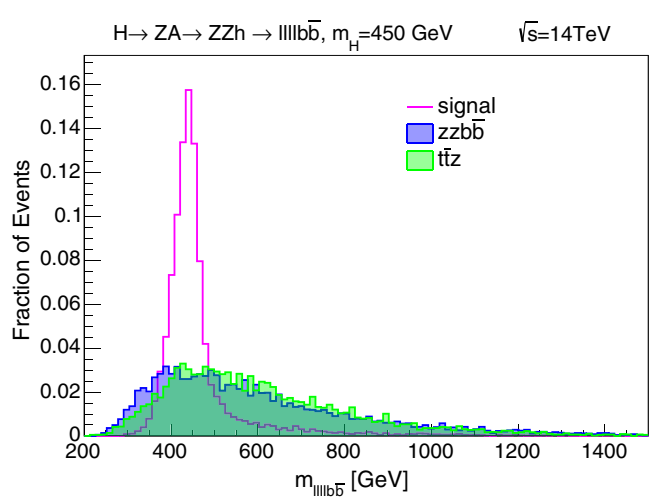

(b) Invariant mass distributions of $\ell^{+} \ell^{-} \ell^{+} \ell^{-} b \bar{b}$. All normalized to 1 .

FIG. 3. Examples of kinematic variables studied for the decay channel $\ell^{+} \ell^{-} \ell^{+} \ell^{-} b \bar{b}$. For the signal, both variables displayed here show prominent features that can be used to suppress the backgrounds.

$b \bar{b}$ and $\ell^{+} \ell^{-} \ell^{+} \ell^{-} b \bar{b}$ for both signal and total background is presented in Fig. 3. The optimized selection requirements based on reconstructed invariant mass of the intermediate bosons are as follows:

(i) $m(b \bar{b}) \in[70,160] \mathrm{GeV}$

(ii) $m\left(\ell^{+} \ell^{-}\right) \in[71,111] \mathrm{GeV}$ for two $\ell^{+} \ell^{-}$pairs.

(iii) $m\left(\ell^{+} \ell^{-} b \bar{b}\right) \in[210,290] \mathrm{GeV}$ for at least one $\ell^{+} \ell^{-}$pair.

(iv) $m\left(\ell^{+} \ell^{-} \ell^{+} \ell^{-} b \bar{b}\right) \in[400,500] \mathrm{GeV}$

Table II displays the number of events after each cut for both signal and backgrounds. The expected significance for our benchmark model in this channel is $11 \sigma$. The integrated luminosity required to reach $5 \sigma$ is $66 \mathrm{fb}^{-1}$, and $3 \sigma$ is $24 \mathrm{fb}^{-1}$.

$$
\text { C. } \boldsymbol{Z Z h} \rightarrow\left(\boldsymbol{\ell}^{+} \boldsymbol{\ell}^{-}\right)(\boldsymbol{j} \boldsymbol{j})(\boldsymbol{b} \overline{\boldsymbol{b}})
$$

This channel benefits from the large branching ratios of $H \rightarrow b \bar{b}$ and $Z \rightarrow j j$, but has significant $t \bar{t} j j$ and $Z b \bar{b} j j$ background. Additional backgrounds such as $W^{+} W^{-}+$ jets and $Z+$ jets, where two jets are misidentified as originating from a $b$-quark, were found to be negligible compared to the two primary backgrounds.

TABLE II. Cut flows after each selection for the $\ell^{+} \ell^{-} \ell^{+} \ell^{-} b \bar{b}$ channel. The number of signal and backgrounds are estimated for a luminosity of $300 \mathrm{fb}^{-1}$. The third column gives the number of events after requiring 2 OSSF lepton pairs and $2 b$-tagged jets.

\begin{tabular}{lccccccc}
\hline \hline Number & 0 & 1 & 2 & 3 & 4 & 5 & 6 \\
\hline Selection & Initial & $\ell^{+} \ell^{-} \ell^{+} \ell^{-} b \bar{b}$ & $m_{b \bar{b}}$ & $m_{\ell^{+} \ell^{-}}$ & $\mathscr{E}_{\mathrm{T}}$ & $m_{A}$ & $m_{H}$ \\
\hline Signal & 353 & 39.5 & 33.6 & 31.8 & 29.5 & 28.4 & 23.9 \\
\hline$t t Z$ & 643 & 37.5 & 14.3 & 3.77 & 1.51 & 1.20 & 0.53 \\
$Z Z b \bar{b}$ & 81.6 & 6.09 & 2.18 & 2.06 & 1.70 & 1.16 & 0.43 \\
$\begin{array}{l}\text { Total } \\
\text { background }\end{array}$ & 725 & 43.6 & 16.5 & 5.83 & 3.21 & 2.35 & 0.96 \\
\hline \hline
\end{tabular}

We require two $b$-tagged jets, two non- $b$-tagged jets, and an OSSF lepton pair. To ensure that the trigger is nearly $100 \%$ efficient, the leading lepton must have $p_{\mathrm{T}}>30 \mathrm{GeV}$.

To devise selection requirements which optimally separate the cascade Higgs signal from SM background, several kinematic variables were considered, including the $p_{\mathrm{T}}$ of the $b \bar{b}$ system and its invariant mass; the $p_{\mathrm{T}}$ of the jet pair and its invariant mass; the $p_{\mathrm{T}}$ of the $\ell^{+} \ell^{-}$system and its invariant mass; the missing transverse energy; and the reconstructed masses of $A$ and $H$. Among all the variables, $m_{b \bar{b}}, m_{\ell^{+} \ell^{-}}, E_{\mathrm{T}}$, $m_{A}$ and $m_{H}$ show the most promise for rejecting SM background. We plot the $m_{b \bar{b}}$ and $m_{\ell^{+} \ell^{-} j j b \bar{b}}$ distributions in Figs. 4(a) and 4(b).

Because the background yields are much larger than the signal, the approximation $S / \sqrt{B}$ is used to calculate the significance. The optimized selection requirements based on reconstructed invariant mass and $E_{\mathrm{T}}$ are as follows:

(i) $m(b \bar{b}) \in[85,160] \mathrm{GeV}$

(ii) $m\left(\ell^{+} \ell^{-}\right) \in[81,101] \mathrm{GeV}$

(iii) $m(j j)>60 \mathrm{GeV}$

(iv) $\mathscr{E}_{\mathrm{T}}<120 \mathrm{GeV}$

(v) Either $m\left(\ell^{+} \ell^{-} b \bar{b}\right)<390 \mathrm{GeV}$ or $m(j j b \bar{b})<390 \mathrm{GeV}$ The event yields for each background and the signal are given in Table III. The significance for this decay mode is $3.6 \sigma$ after the invariant mass selections on the candidate $A$ and candidate $H$.

In this channel, the background yield is much larger than the signal. So unlike the other channels, the uncertainty of the background may largely affect the outcome. In the calculation, the background uncertainty is set to be 5\% for cross section and $3 \%$ for luminosity. By using likelihood ratio (LR) method, instead of simple $S / \sqrt{B}$, we calculate the significance to be $2.2 \sigma$. Moreover, by using boosted decision tree (BDT) method, we find that the signal significance could reach 5.2 $\sigma$. In this method, the same five variables previously used in the cut-and-count analysis and $m_{\ell^{+} \ell^{-} j j b \bar{b}}$ constitute the input of the BDT. The output, 


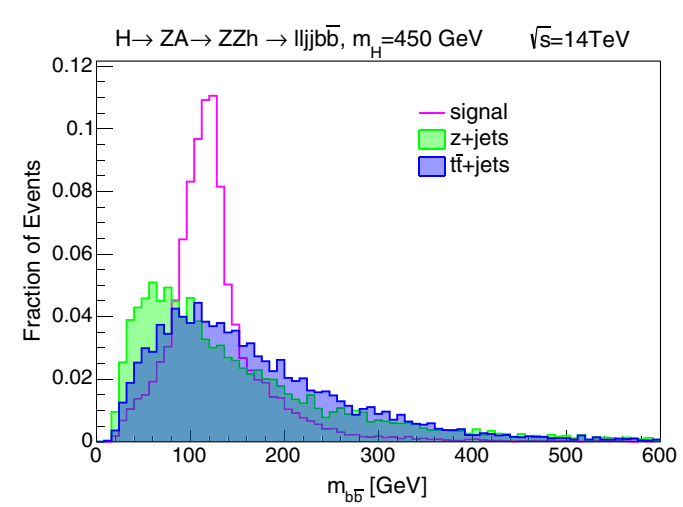

(a) Di-Bottom invariant mass distributions, normalized to one.

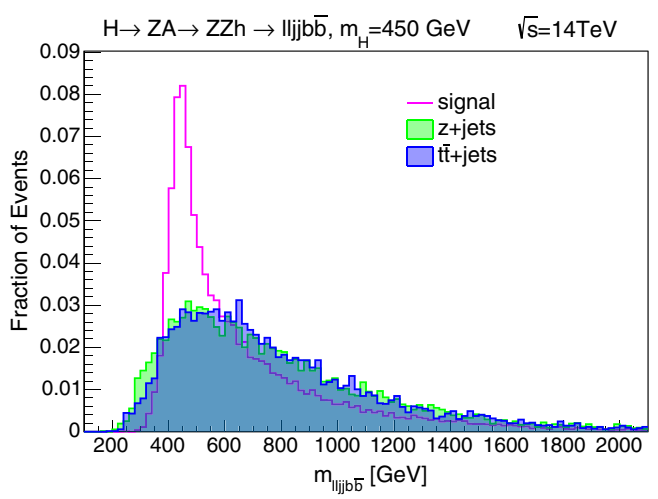

(b) $\ell^{+} \ell^{-} j j b \bar{b}$ invariant mass distributions, normalized to one.

FIG. 4. Examples of kinematic variables studied for the decay channel $\ell^{+} \ell^{-} j j b \bar{b}$.

the BDT response, is used to calculate the significance with LR method, see Fig. 5.

To demonstrate that our results are robust to uncertainties in the background, we plot the signal significance as a function of the fractional background uncertainty for both the LR and the BDT methods (Fig. 6). As can be seen, the fluctuation in background uncertainties does not significantly affect the signal significance.

\section{D. $Z Z h \rightarrow \ell^{+} e^{-} e^{+} e^{-}+E_{\mathrm{T}}$}

In this channel, the relatively small decay branching ratios involved are partially compensated by the large number of combinations of decays which result in this final state. The main contribution is from cases where at least one $Z$ boson decays as $Z \rightarrow \ell^{+} \ell^{-}$while the Higgs decays either as $h \rightarrow \ell \nu \ell \nu$ or $h \rightarrow \tau_{\ell} \tau_{\ell}$ or the other $Z \rightarrow$ $\tau_{\ell} \tau_{\ell}$ where $\tau_{\ell}$ denotes a $\tau$ which subsequently decays leptonically.

The primary SM backgrounds are as follows:

(i) $Z Z$ production, with each $Z$ decaying either as $Z \rightarrow$ $\ell^{+} \ell^{-}$or $Z \rightarrow \tau_{\ell} \tau_{\ell}$.

(ii) SM Higgs boson production with $h \rightarrow 4 \ell$;

(iii) $Z h$ production with $Z \rightarrow \ell^{+} \ell^{-}$and $h \rightarrow \ell^{+} \ell^{-} \nu \nu$ or $Z \rightarrow \nu \nu$ and $h \rightarrow 4 \ell$

(iv) $W h$ production with $W \rightarrow l \nu$ and $h \rightarrow 4 \ell$.

The leading lepton must have $p_{\mathrm{T}}>20 \mathrm{GeV}$ and the subleading lepton $p_{\mathrm{T}}>15 \mathrm{GeV}$. In addition, at least one OSSF lepton pair is required to have a reconstructed invariant mass in the range $81 \mathrm{GeV}<m\left(\ell^{+} \ell^{-}\right)<$ $101 \mathrm{GeV}$. The signal processes have larger $E_{\mathrm{T}}$ than most of the SM background, as shown in Fig. 7, and the selection requirement which maximizes the signal significance was found to be $E_{\mathrm{T}}>150 \mathrm{GeV}$.

Table IV lists the number of signal and background events obtained after the final cuts. This significance of the benchmark model after all cuts is $2.1 \sigma$.

$$
\text { E. } \boldsymbol{Z Z h} \rightarrow\left(\boldsymbol{\ell}^{+} \boldsymbol{\ell}^{-}\right)(\boldsymbol{\nu} \boldsymbol{\nu})(\boldsymbol{\gamma} \boldsymbol{\gamma})
$$

The main SM backgrounds for this channel are $t \bar{t} \gamma j$ (where $j$ is misidentified as a photon), $t \bar{t} \gamma \gamma, W Z \gamma \gamma, \ell^{+} \ell^{-} \nu \bar{\nu} \gamma \gamma$ and $Z \gamma \gamma$. We also considered $\tau^{+} \tau^{-} \gamma \gamma$ with the $\tau^{\prime} s$ decaying leptonically, but it was completely negligible after applying invariant mass cuts. For the $t \bar{t} \gamma+$ jets background, we use $10^{-3}$ for the probability of a jet faking a photon, which is consistent with the CMS detector performance [20]. For the $Z \gamma \gamma$ and the $\ell^{+} \ell^{-} \nu \bar{\nu} \gamma \gamma$ backgrounds, we also take into account the contribution from $h \rightarrow \gamma \gamma$.

Despite of a very clean signature, this channel suffers from very small cross sections for both the signal and backgrounds. We have applied $p_{\mathrm{T}}$ cuts for the photons and leptons, invariant mass cuts for the two leading charged leptons and photons, and a MET cut to take advantage of the neutrinos in the final state. After optimizing the cuts for the significance, the final selections are as follows:

TABLE III. Cut flows after each selection for the $\ell^{+} \ell^{-} j j b \bar{b}$ channel. The number of signal and backgrounds are estimated for a luminosity of $300 \mathrm{fb}^{-1}$. The third column gives the number of events after requiring an OSSF lepton pair, $2 b$-tagged jets and 2 jets.

\begin{tabular}{lccccccc}
\hline \hline Number & 0 & 1 & 2 & 3 & 4 & 5 & 6 \\
\hline Selection & Initial & $\ell^{+} \ell^{-} j j b \bar{b}$ & $m_{b \bar{b}}$ & $m_{\ell^{+} \ell^{-}}$ & $m_{j j}$ & $E_{\mathrm{T}}$ \\
\hline Signal & 5660 & 916 & 606 & 560 & 526 & 510 \\
\hline$Z+$ jets & $7.1 \times 10^{6}$ & $8.8 \times 10^{4}$ & $2.3 \times 10^{4}$ & $2.2 \times 10^{4}$ & $1.8 \times 10^{4}$ & $1.7 \times 10^{4}$ & $1.4 \times 10^{4}$ \\
$t \bar{t} j j$ & $2.7 \times 10^{7}$ & $2.5 \times 10^{5}$ & $7.3 \times 10^{4}$ & $1.0 \times 10^{4}$ & $8.5 \times 10^{3}$ & $5.9 \times 10^{3}$ & $5.7 \times 10^{3}$ \\
Total background & $3.4 \times 10^{7}$ & $3.4 \times 10^{5}$ & $9.6 \times 10^{4}$ & $3.2 \times 10^{4}$ & $2.6 \times 10^{4}$ & $2.3 \times 10^{4}$ & $1.9 \times 10^{4}$ \\
\hline \hline
\end{tabular}




\section{TMVA response for classifier: BDT}

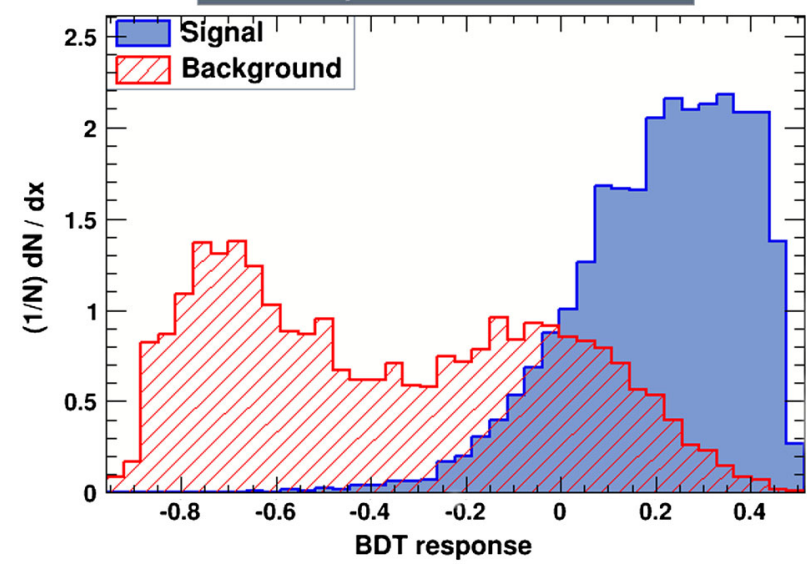

FIG. 5. The BDT response distribution with input variables of $m_{b \bar{b}}, m_{\ell^{+} \ell^{-}}, m_{j j}, E_{\mathrm{T}}, m_{A}$ and $m_{H}$.

(i) Two photons with $p_{\mathrm{T}}>17 \mathrm{GeV}$

(ii) Two leptons with $p_{\mathrm{T}}>13 \mathrm{GeV}$

(iii) Missing $E_{T}>50 \mathrm{GeV}$

(iv) Invariant mass $m_{\gamma \gamma} \in[120,130] \mathrm{GeV}$

(v) Invariant mass $m_{\ell^{+} \ell^{-}} \in[84,98] \mathrm{GeV}$

Table $\mathrm{V}$ lists the number of events obtained after the final selection for the signal and the backgrounds. Despite of a sharp drop in the signal cross section due to the tiny $h \rightarrow \gamma \gamma$ branching ratio $\left(\sim 10^{-3}\right)$, the good resolution in the reconstructed $M_{\gamma \gamma}$ is especially effective in suppressing the backgrounds. The significance of this channel was found to be $2.8 \sigma$, provided that the signal yields is 2.1 and that the background yields is 0.14 .

\section{F. $\boldsymbol{Z Z h} \rightarrow\left(\boldsymbol{\ell}^{+} \boldsymbol{\ell}^{-}\right)(\boldsymbol{j j})(\boldsymbol{\gamma} \boldsymbol{\gamma})$}

This channel faces the similar challenges as the $\ell^{+} \ell^{-} \nu \nu \gamma \gamma$ channel, where both the signal and the background cross sections are fairly small. However, the sharp resolution in $M_{\text {inv }}(\gamma \gamma)$ for the signal helps significantly

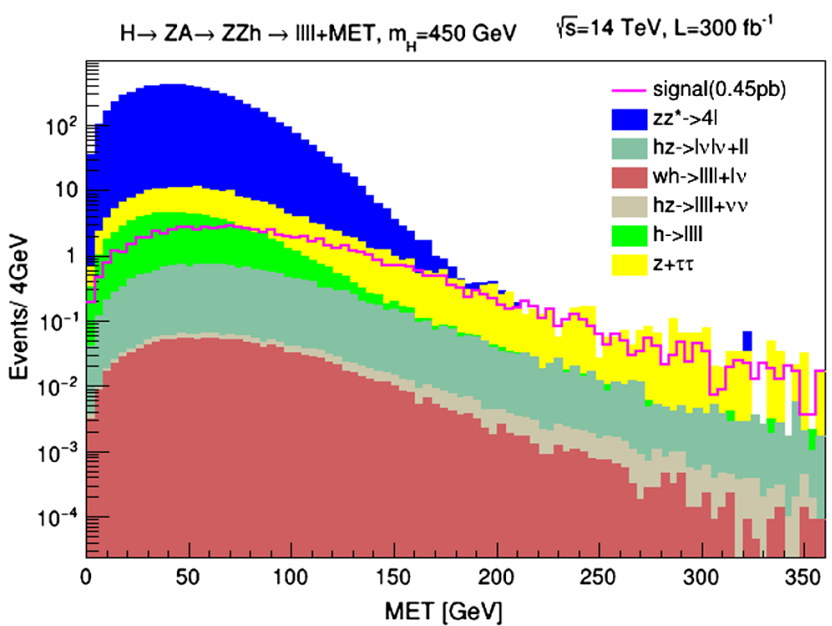

FIG. 7. $E_{\mathrm{T}}$ distribution for signal and backgrounds after 4 lepton selection with one OSSF at Z boson mass resonance. A high $E_{\mathrm{T}}$ cut is favored as can be seen. An integrated luminosity of $300 \mathrm{fb}^{-1}$ is assumed.

suppress the backgrounds. The main SM backgrounds for this channel contain the irreducible background $\ell^{+} \ell^{-} j j \gamma \gamma$, $\ell^{+} \ell^{-} \gamma+$ jets with one jet-faked photon and $\ell^{+} \ell^{-}+$jets with two fake photons. Other SM processes, such as $t \bar{t}+$ fake $\gamma \mathrm{s}, t \bar{t} \gamma \gamma$ and $Z Z \gamma+$ fake $\gamma$ can also contribute but their final yields are much smaller compared to the main backgrounds due to their smaller cross sections and the tiny jet-faking-photon rate. Similarly to the $Z \rightarrow j j$ decay in the $\ell^{+} \ell^{-} j j b \bar{b}$ final state search, the reconstructed $m_{j j}$ has so broad a peak that it cannot be exploited. The final selections are as follows:

(i) Two photons with $p_{\mathrm{T}}>24 \mathrm{GeV}$

(ii) Two jets with $p_{\mathrm{T}}>24 \mathrm{GeV}$

(iii) Two leptons with $p_{\mathrm{T}}>13 \mathrm{GeV}$

(iv) Invariant mass $m_{\gamma \gamma} \in[120,130] \mathrm{GeV}$

(v) Invariant mass $m_{\ell^{+} \ell^{-}} \in[81,101] \mathrm{GeV}$

(vi) Missing $E_{T}<120 \mathrm{GeV}$

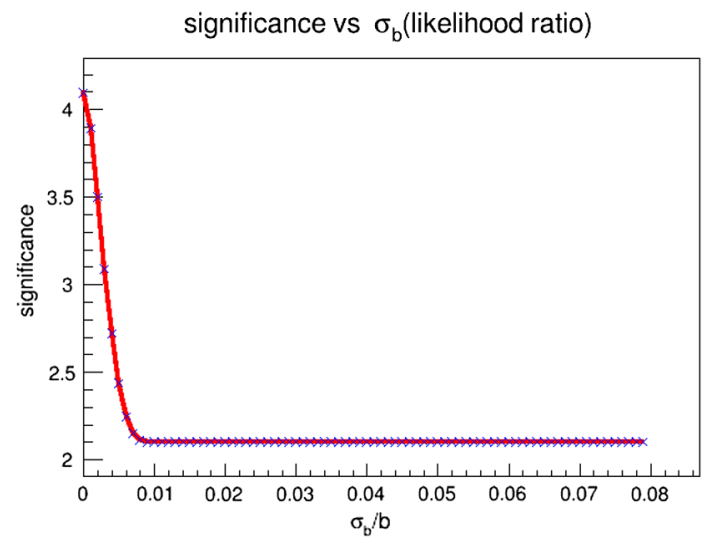

(a)

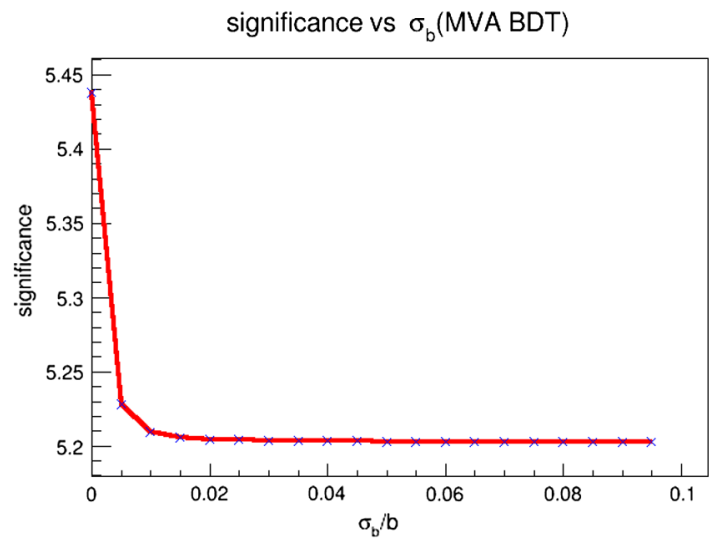

(b)

FIG. 6. The signal significance as a function of the fractional background uncertainty. 
TABLE IV. The event yields for the signal and backgrounds after the selection for the $\ell^{+} \ell^{-} \ell^{+} \ell^{-}+E_{\mathrm{T}}$ channel. An integrated luminosity of $300 \mathrm{fb}^{-1}$ is assumed.

\begin{tabular}{lcc}
\hline \hline \multirow{2}{*}{ Signal } & Initial & Final Yields \\
\cline { 2 - 3 }$Z Z \rightarrow\left(\ell^{+} \ell^{-}\right)\left(\ell^{+} \ell^{-}\right)$ & 1600 & 9.7 \\
\hline$Z \rightarrow\left(\tau^{+} \tau^{-}\right)\left(\ell^{+} \ell^{-}\right)$ & $1.5 \times 10^{4}$ & 8.2 \\
$h \rightarrow \ell^{+} \ell^{-} \ell^{+} \ell^{-}$ & 7700 & 8.4 \\
$Z h \rightarrow\left(\ell^{+} \ell^{-}\right)\left(\ell^{+} \ell^{-} \nu \nu\right)$ & 240 & 0.16 \\
$Z h \rightarrow(\nu \nu)\left(\ell^{+} \ell^{-} \ell^{+} \ell^{-}\right)$ & 41 & 1.3 \\
$W h \rightarrow(\ell \nu)\left(\ell^{+} \ell^{-} \nu \nu\right)$ & 1.3 & 0.08 \\
Total background & 2.3 & 0.09 \\
\hline \hline
\end{tabular}

Table VI shows the cut flows and signal and background yields for this channel. With 5.7 signal events and 9.6 background events, we get a $1.7 \sigma$ significance.

\section{G. Other Channels}

There exist other channels that do not perform as well as the previously described 5 channels, nevertheless show interesting features that are worth considering given the prospect of a higher integrated luminosity.

One of them is $Z \rightarrow \nu \nu, Z \rightarrow j j, h \rightarrow \gamma \gamma$. As in the case of $\ell^{+} \ell^{-} j j b \bar{b}$ [Fig. 4(b)], the reconstructed $Z$ from dijet is
TABLE VII. Cut flows for the $\gamma \gamma \nu \nu j j$ channel. An integrated luminosity of $300 \mathrm{fb}^{-1}$ is assumed.

\begin{tabular}{lccccc}
\hline \hline Number & 0 & 1 & 2 & 3 & 4 \\
\hline Selection & Initial & $j j \gamma \gamma$ & $m_{\gamma \gamma}$ & $m_{j j}$ & $E_{\mathrm{T}}$ \\
\hline Signal & 67.8 & 38.9 & 28.6 & 27.4 & 7.4 \\
\hline $\begin{array}{l}\text { Total } \\
\text { background }\end{array}$ & $3.8 \times 10^{12}$ & $2.6 \times 10^{7}$ & $3.3 \times 10^{5}$ & $2.9 \times 10^{5}$ & $2.4 \times 10^{2}$ \\
\hline \hline
\end{tabular}

almost useless in differentiating between the signal and the backgrounds. Therefore, this channel suffers not only from a small cross section, but also from "wasting" two Zs. However, just as those channels with $h \rightarrow \gamma \gamma$, the outstanding resolution in the reconstructed $m_{h}$ can be very powerful in suppressing the background. The event selection requires two photons that satisfy $122<m_{\gamma \gamma}<$ $128 \mathrm{GeV}$, two jets that satisfy $m_{j j}>60 \mathrm{GeV}$ and a $E_{\mathrm{T}}$ requirement of $E_{\mathrm{T}}>140 \mathrm{GeV}$. Due to the massive cross section, QCD production of two photons still dominates after the selection of $m_{h}$ and high $E_{\mathrm{T}}$ requirement. The final signal yield is 7.4 while background yield is 240 , giving a significance of $0.5 \sigma$ assuming an integrated luminosity of $300 \mathrm{fb}^{-1}$. Table VII displays the cut flows for the signal and the total background for this channel.

TABLE V. Cut flows for the $\gamma \gamma \nu \nu \ell^{+} \ell^{-}$channel. An integrated luminosity of $300 \mathrm{fb}^{-1}$ is assumed. The third column gives the number of events obtained after requiring an OSSF lepton pair and two photons.

\begin{tabular}{lcccccc}
\hline \hline Number & 0 & 1 & 2 & 3 & 4 & 5 \\
\hline Selection & Initial & $\ell^{+} \ell^{-} \gamma \gamma$ & $p_{T_{\ell, \gamma}}$ & $m_{\gamma \gamma}$ & $m_{\ell^{+} \ell^{-}}$ & $E_{\mathrm{T}}$ \\
\hline Signal & 8.3 & 3.3 & 3.1 & 2.8 & 2.5 & 2.1 \\
\hline$t \bar{t} \gamma+$ jets $(1$ fake $\gamma)$ & 41 & 10 & 6.8 & 0.27 & 0.078 & 0.059 \\
$Z \gamma \gamma$ & 106 & 56 & 46 & 20 & 18 & 0.028 \\
$t \bar{t} \gamma \gamma$ & 2.5 & 0.51 & 0.39 & 0.13 & 0.038 & 0.028 \\
$\ell^{+} \ell^{-} \nu \bar{\nu} \gamma \gamma$ & 0.48 & 0.25 & 0.19 & 0.078 & 0.035 & 0.024 \\
$W Z \gamma \gamma$ & 0.064 & 0.041 & 0.030 & $9.1 \times 10^{-3}$ & $5.1 \times 10^{-3}$ & $2.4 \times 10^{-3}$ \\
Total background & 150 & 67 & 43 & 20 & 18 & 0.14 \\
\hline \hline
\end{tabular}

TABLE VI. Cut flows for the $\gamma \gamma j j \ell^{+} \ell^{-}$channel. An integrated luminosity of $300 \mathrm{fb}^{-1}$ is assumed.

\begin{tabular}{|c|c|c|c|c|c|c|}
\hline Number & 0 & 1 & 2 & 3 & 4 & 5 \\
\hline Selection & Initial & $\gamma \gamma j j \ell^{+} \ell^{-}$ & $p_{T_{\ell, \gamma, j}}$ & $m_{\gamma \gamma}$ & $m_{\ell^{+} \ell^{-}}$ & $E_{\mathrm{T}}$ \\
\hline Signal & 29 & 8.3 & 6.8 & 6.2 & 5.7 & 5.7 \\
\hline$\overline{\ell^{+} \ell^{-} j j \gamma \gamma}$ & 230 & 38 & 18 & 9.4 & 7.1 & 7.1 \\
\hline$\ell^{+} \ell^{-} \gamma j j j(1$ fake $\gamma)$ & 12900 & 267 & 66 & 2.6 & 1.9 & 1.9 \\
\hline$\ell^{+} \ell^{-}+$jets $(2$ fake $\gamma \mathrm{s})$ & 4640 & 24 & 14 & 0.53 & 0.48 & 0.48 \\
\hline$t \bar{t} \gamma j(1$ fake $\gamma)$ & 25 & 4.0 & 2.5 & 0.11 & 0.054 & 0.043 \\
\hline$t \bar{t} \gamma \gamma$ & 1.1 & 0.20 & 0.13 & 0.062 & 0.031 & 0.024 \\
\hline$t \bar{t}+$ jets $(2$ fake $\gamma \mathrm{s})$ & 19 & 2.1 & 1.6 & 0.055 & 0.027 & 0.022 \\
\hline$Z Z \gamma j$ (1 fake $\gamma)$ & 1.4 & 0.52 & 0.32 & 0.013 & 0.012 & 0.012 \\
\hline Total background & 17800 & 335 & 103 & 13 & 9.6 & 9.6 \\
\hline
\end{tabular}


TABLE VIII. Cut flows for the $\ell^{+} \ell^{-} j j \tau_{h} \tau_{h}$ channel. An integrated luminosity of $300 \mathrm{fb}^{-1}$ is assumed.

\begin{tabular}{lccccccc}
\hline \hline Number & 0 & 1 & 2 & 3 & 4 & 5 & 6 \\
\hline Selection & Initial & $\ell^{+} \ell^{-} j j \tau \tau$ & $m_{\tau \tau}$ & $m_{\ell^{+} \ell^{-}}$ & $E_{\mathrm{T}}$ & $m_{A}$ & $m_{H}$ \\
\hline Signal & 630 & 26 & 18 & 17 & 17 & 15 & 11 \\
\hline Total background & $2.3 \times 10^{6}$ & $4.9 \times 10^{3}$ & $1.3 \times 10^{3}$ & $1.3 \times 10^{3}$ & $1.2 \times 10^{3}$ & 620 & 290 \\
\hline \hline
\end{tabular}

TABLE IX. Summary of the most sensitive channels of the $H \rightarrow Z A, A \rightarrow Z h$ cascade search for a benchmark $m_{H}=450 \mathrm{GeV}$, $m_{A}=m_{H^{ \pm}}=250 \mathrm{GeV}$, assuming the $Z Z h$ production cross section is $0.45 \mathrm{pb}$ for the signal.

\begin{tabular}{lccccccc}
\hline \hline & \multicolumn{3}{c}{$14 \mathrm{TeV} 300 \mathrm{fb}^{-1}$} & & \multicolumn{3}{c}{$14 \mathrm{TeV} 3000 \mathrm{fb}^{-1}$} \\
\cline { 2 - 4 } Channel & Sig Yields & Bkg Yields & Significance & & Sig Yields & Bkg Yields & Significance \\
\hline$\ell^{+} \ell^{-} \ell^{+} \ell^{-} b \bar{b}$ & 24 & 0.96 & $11 \sigma$ & & 240 & 9.6 & $34 \sigma$ \\
$\ell^{+} \ell^{-} j j b \bar{b}$ & 495 & $1.9 \times 10^{4}$ & $3.6 \sigma$ & & 4950 & $1.9 \times 10^{5}$ & $11 \sigma$ \\
$\ell^{+} \ell^{-} \ell^{+} \ell^{-}+E_{\mathrm{T}}^{+}$ & 9.7 & 18 & $2.1 \sigma$ & & 97 & 180 & $6.6 \sigma$ \\
$\ell^{+} \ell^{-} \nu \nu \gamma \gamma$ & 2.1 & 0.14 & $2.8 \sigma$ & & 21 & 1.4 & $9.1 \sigma$ \\
$\ell^{+} \ell^{-} j j \gamma \gamma$ & 5.7 & 9.6 & $1.7 \sigma$ & & 57 & 96 & $5.3 \sigma$ \\
\hline \hline
\end{tabular}

Another interesting decay mode is $Z \rightarrow \ell^{+} \ell^{-}, Z \rightarrow j j$, $h \rightarrow \tau_{h} \tau_{h}$. This channel utilizes the hadronic tau tagging in LHC detectors. Compared to $Z \rightarrow \ell^{+} \ell^{-}, Z \rightarrow j j, h \rightarrow b \bar{b}$, it successfully avoids the large background from $t \bar{t}+$ jets. But it is seriously inflicted by the high tau mistagging rate. Hence, $Z+$ jets with two misidentified $\tau_{h}$ s dominates. The event selection requires an OSSF lepton pair that satisfies $76<m_{\ell^{+} \ell^{-}}<106 \mathrm{GeV}$, an OSSF tau pair that satisfies $80<m_{\tau_{h} \tau_{h}}<150 \mathrm{GeV}$, two non-tau-tagged jets, $E_{\mathrm{T}}<130 \mathrm{GeV}, \quad m_{A}<330 \mathrm{GeV}$ and $m_{H}<670 \mathrm{GeV}$. $m_{\ell^{+} \ell^{-}}$and $E_{\mathrm{T}}$ selection is applied to ensure that the other SM backgrounds are negligible compared to DY lepton pair production. The final significance is about $0.7 \sigma$. Table VIII gives the cut flows for the signal and the backgrounds.

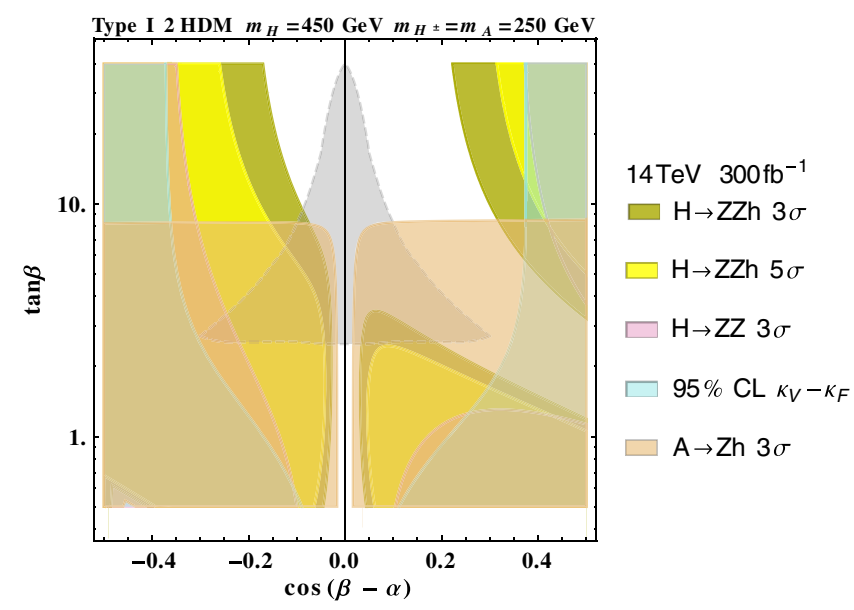

FIG. 8. Projections of the $A$ and $H$ direct searches and the SM Higgs coupling measurements for a Type I $2 \mathrm{HDM}$ at LHC run II, assuming $m_{H}=450 \mathrm{GeV}, m_{A}=m_{H^{ \pm}}=250 \mathrm{GeV}$. Constraints from $h \rightarrow b b, \tau \tau$ are much weaker for large $\tan \beta$ and are not included.

\section{H. Results of the benchmark study}

In Table IX, we list the number of signal and background yields for each channel studied and the significance reached at both $300 \mathrm{fb}^{-1}$ and $3000 \mathrm{fb}^{-1}$.

Among all the channels studied, $\ell^{+} \ell^{-} \ell^{+} \ell^{-} b \bar{b}$ performs the best; the four-lepton requirement provides a clean signal, and $h \rightarrow b \bar{b}$ has a relatively large branching ratio. The next best performing channel is $\ell^{+} \ell^{-} j j b \bar{b}$. Although $\ell^{+} \ell^{-} j j b \bar{b}$ gives the most signal events, it is hard to suppress the $Z+$ jets backgrounds, for the jet shower is severe, the resolution of jet energy is not as good as that of leptons, and the reconstructed dijet mass peak is not significant enough to suppress the backgrounds. $\ell^{+} \ell^{-} \nu \nu \gamma \gamma$ and $\ell^{+} \ell^{-} j j \gamma \gamma$ enjoy an outstanding resolution in the reconstructed $m_{h}$, meanwhile suffering from a much smaller cross section compared to the three other channels.

For the most sensitive channel $\ell^{+} \ell^{-} \ell^{+} \ell^{-} b \bar{b}$, we compare the significance reach of this channel with those of the direct $A$ and $H$ searches at $14 \mathrm{TeV}$ for our benchmark. For simplicity, we only consider $A \rightarrow Z h \rightarrow \ell^{+} \ell^{-} b \bar{b}$ and $H \rightarrow$ $Z Z \rightarrow \ell^{+} \ell^{-} \ell^{+} \ell^{-}$for the direct searches, both of which were studied by CMS and ATLAS in LHC Run I. Details of the direct searches considered here can be found in Appendix B and C.

In Fig. 8, we give projections of the $A$ and $H$ direct searches, assuming an integrated luminosity of $300 \mathrm{fb}^{-1}$. We also display the ATLAS' projections of 95\% CL likelihood contours for $\kappa_{V}$ and $\kappa_{F}{ }^{1}$ at $14 \mathrm{TeV}$ with $300 \mathrm{fb}^{-1}[21]$ in the same plot. In Fig. 8, the limits that can be reached by the $H$ cascade search are shown side by

\footnotetext{
${ }^{1} \kappa_{F}\left(\kappa_{V}\right)$ is the ratio between the Higgs to fermions (vector bosons) coupling to its $\mathrm{SM}$ value.
} 


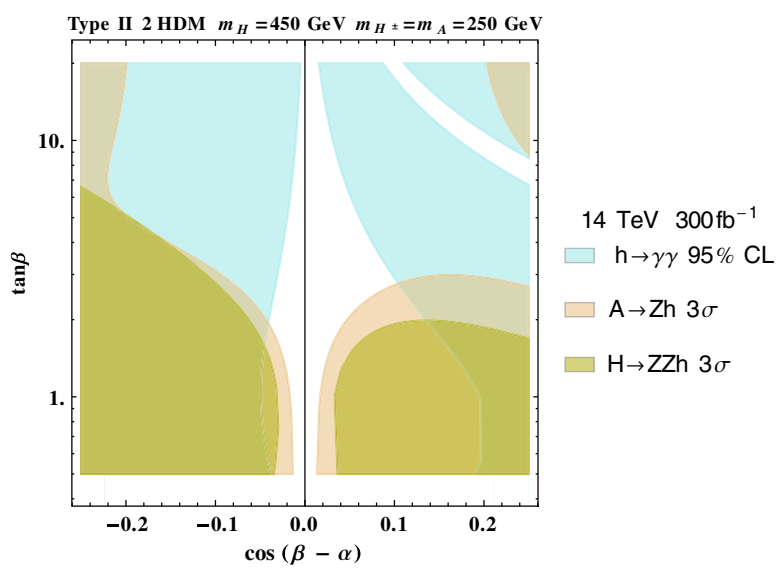

FIG. 9. The projections of the $\operatorname{direct} A$ search, the $H$ cascade search and the ATLAS's $h \rightarrow \gamma \gamma$ coupling measurement projected at LHC run II, for $m_{H}=450 \mathrm{GeV}, m_{A}=m_{H^{ \pm}}=250 \mathrm{GeV}$.

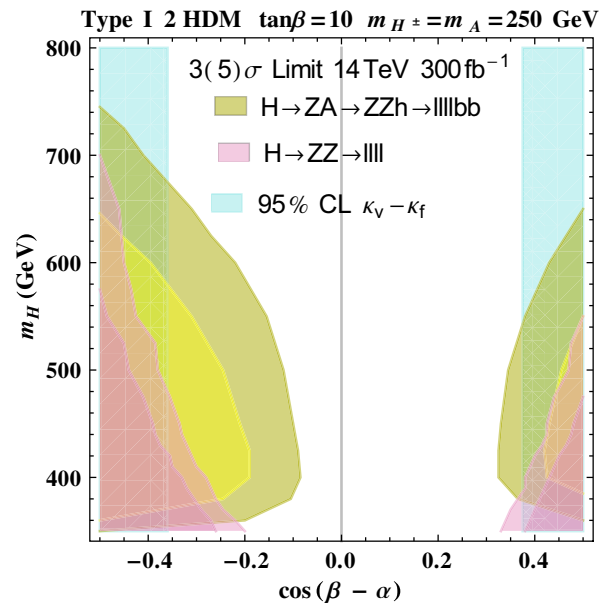

FIG. 10. The limits that can be reached by the $H$ cascade search as a function of its mass with fixed $\tan \beta, m_{A}$ and $m_{H^{ \pm}}$, assuming an integrated luminosity of $300 \mathrm{fb}^{-1}$. For comparison, the limits are also plotted for the direct $H$ search and the ATLAS's projections on SM Higgs coupling measurements.

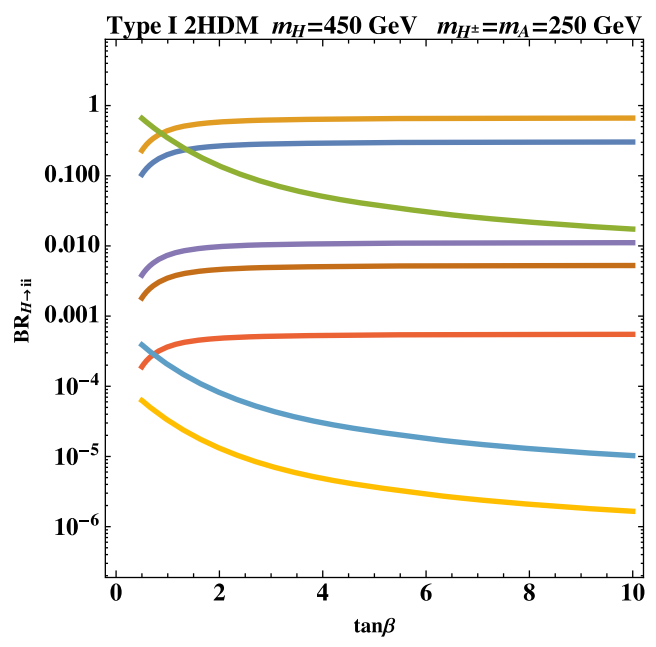

(a) $\cos (\beta-\alpha)=-0.1$. side with the direct searches. It can be clearly seen that the $\ell^{+} \ell^{-} \ell^{+} \ell^{-} b \bar{b}$ channel from the $H$ cascade search is more sensitive for $\tan \beta \gtrsim 8$.

We show the sensitivity of our search for a type II model in Fig. 9. We compare the significance reach of the $\ell^{+} \ell^{-} \ell^{+} \ell^{-} b \bar{b}$ channel from the $H$ cascade search with the direct search $A \rightarrow Z h \rightarrow \ell^{+} \ell^{-} b \bar{b}$ for our benchmark. We also display the ATLAS' projection on $h \rightarrow \gamma \gamma$ cross section at $14 \mathrm{TeV}$ in the same plot. As one can see, our search has comparable sensitivity to the direct $A \rightarrow Z h$ search, but the latter is slightly more sensitive.

The benchmark study shows that the $H$ cascade search is most sensitive at large $\tan \beta$. We consider as a benchmark $\tan \beta=10$, and investigate the range in $m_{H}$ for which this search provides new sensitivity. For $\tan \beta=10$, the direct $A$ search is completely ineffective (see Fig. 8) because the production of $A$ via quark loops is suppressed by $\tan ^{2} \beta$ in Type I 2HDM. That leaves the direct $H$ search as the only relevant comparison to the $H$ cascade search for this benchmark. As shown in Fig. 11(b), in the channels that $H$ directly decays to the SM particles, $H \rightarrow W W$ and $H \rightarrow Z Z$ are dominant. Since the channel $H \rightarrow Z Z \rightarrow 4 \ell$ benefits from a relatively clean background and high efficiencies of the lepton ID, it allows a full reconstruction of the decay chain. Therefore, $H \rightarrow Z Z \rightarrow 4 \ell$ is the most sensitive channel among all the direct $H$ searches. As can be seen from Fig. 10, the $3 \sigma$ exclusion of the $H$ cascade search reaches $m_{H} \sim 650 \mathrm{GeV}$ within two standard deviations of the SM limit. The direct $H$ search is not as sensitive as the cascade search, as it suffers from the SM ZZ background.

The theory makes no prediction for the value of $m_{H}$, and therefore the look-elsewhere effect should be considered. However, considering the optimized selection criteria for $m_{\ell^{+} \ell^{-} \ell^{+} \ell^{-} b \bar{b}}$ in the most sensitive channel, we estimate that the number of independent $m_{H}$ bins in the region this analysis is sensitive is only about

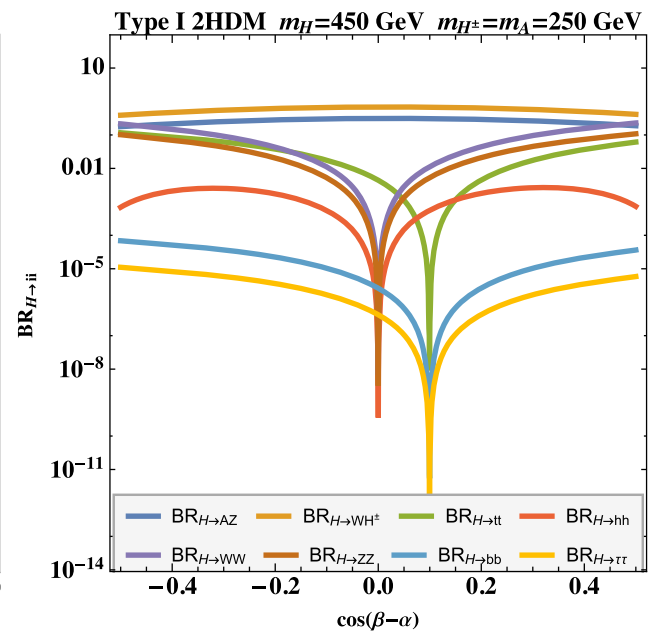

(b) $\tan \beta=10$.

FIG. 11. Branching Ratios of $H$ in our benchmark model. 
three. Thus, the look-elsewhere effect is not expected to significantly affect the projected sensitivity of this search.

\section{CONCLUSIONS}

In this paper, we demonstrate that the search $g g \rightarrow H \rightarrow$ $A Z \rightarrow Z Z h$ can be a discovery mode for additional Higgs boson at the $14 \mathrm{TeV}$ LHC. For a benchmark with $m_{A}=$ $250 \mathrm{GeV}$ and $m_{H}=450 \mathrm{GeV}$ we find that the significance with $300 \mathrm{fb}^{-1}$ is over $10 \sigma$ in the "golden channel" $\left(\ell^{+} \ell^{-}\right)\left(\ell^{+} \ell^{-}\right)(b \bar{b})$, and that the most signal abundant channel $\left(\ell^{+} \ell^{-}\right)(j j)(b \bar{b})$ may reach $5 \sigma$ significance with a boosted decision tree analysis. At the high-luminosity LHC $\left(3000 \mathrm{fb}^{-1}\right)$ the prospects are even better, with five channels having over $5 \sigma$ significance. We emphasize that this benchmark will not be probed by other search channels or Higgs coupling measurements. The reach also persists for values of $m_{H}$ up to $650 \mathrm{GeV}$, again in models that are not probed by existing searches. We conclude that searches for $Z Z h$ final state are highly motivated at the $14 \mathrm{TeV}$ LHC.

\section{ACKNOWLEDGMENTS}

We are grateful to E. Salvioni and Y. Tsai for their help at early stages of this work. M. A. L. wishes to thank the Galileo Galilei Institute for hospitality. This work was supported by DOE Grant No. DE-FG02-91ER406746.

\section{APPENDIX A: PROPERTIES OF THE BENCHMARK}

Four our study we choose a benchmark model where the masses of all physical Higgs bosons are fixed. We choose $m_{A}=250 \mathrm{GeV}$ so that the decay $A \rightarrow Z h$ is open but the decay $A \rightarrow t \bar{t}$ is closed. We choose $m_{H}=$ $450 \mathrm{GeV}$ so that the decay $H \rightarrow Z A$ is open. These masses are chosen near the lower end of the lowest range for which the cascade decay $H \rightarrow Z A \rightarrow Z Z h$ is allowed in order to maximize the rate. The main constraint from precision electroweak data comes from custodial symmetry breaking [22], and this is suppressed by choosing $m_{H^{ \pm}}=m_{A}$. With these masses fixed, there is still sufficient freedom to suppress the Higgs self-couplings, so that the $H \rightarrow h h$ decay is unimportant. To illustrate this, in Figure 11, the $H$ branching ratios are plotted at a fixed $\cos (\beta-\alpha)$ and $\tan \beta$, respectively.

\section{Theoretical constraints}

To analyze the theoretical constraints on this benchmark model we solve the 2HDM potential parameters in terms of the masses. The Higgs potential is

$$
\begin{aligned}
V= & m_{11}^{2}\left(\phi_{1}^{\dagger} \phi_{1}\right)+m_{22}^{2}\left(\phi_{2}^{\dagger} \phi_{2}\right)+\left[m_{12}^{2}\left(\phi_{1}^{\dagger} \phi_{2}\right)+\text { h.c. }\right] \\
& +\frac{\lambda_{1}}{2}\left(\phi_{1}^{\dagger} \phi_{1}\right)^{2}+\frac{\lambda_{2}}{2}\left(\phi_{2}^{\dagger} \phi_{2}\right)^{2}+\lambda_{3}\left(\phi_{1}^{\dagger} \phi_{1}\right)\left(\phi_{2}^{\dagger} \phi_{2}\right) \\
& +\lambda_{4}\left(\phi_{1}^{\dagger} \phi_{2}\right)\left(\phi_{2}^{\dagger} \phi_{1}\right)+\frac{1}{2}\left[\lambda_{5}\left(\phi_{1}^{\dagger} \phi_{2}\right)^{2}+\text { h.c. }\right],
\end{aligned}
$$

where all the parameters are assumed real. The constraints arise from requiring stability of the potential for large field values and perturbativity of dimensionless couplings. We impose the standard requirements

$$
0<\lambda_{1}<4 \pi, \quad 0<\lambda_{2}<4 \pi, \quad \lambda_{3}>-\sqrt{\lambda_{1} \lambda_{2}} .
$$

We trade the Higgs potential parameters $\lambda_{1}, \lambda_{2}, \lambda_{3}, \lambda_{4}, \lambda_{5}$, $m_{11}^{2}, m_{22}^{2}$ for the phenomenological parameters: $v, \beta, \alpha, m_{h}$, $m_{H}, m_{A}=m_{H^{ \pm}}$:

$$
\begin{aligned}
& \lambda_{1}=\frac{m_{H}^{2}}{v^{2}}\left(c_{\beta-\alpha}+s_{\beta-\alpha} t_{\beta}\right)^{2}+\frac{m_{h}^{2}}{v^{2}}\left(c_{\beta-\alpha} t_{\beta}-s_{\beta-\alpha}\right)^{2}-\frac{2 m_{12}^{2} t_{\beta}^{2}}{s_{2 \beta} v^{2}}, \\
& \lambda_{2}=\frac{m_{H}^{2}}{v^{2}}\left(c_{\beta-\alpha}-\frac{s_{\beta-\alpha}}{t_{\beta}}\right)^{2}+\frac{m_{h}^{2}}{v^{2}}\left(\frac{c_{\beta-\alpha}}{t_{\beta}}+s_{\beta-\alpha}\right)^{2}-\frac{2 m_{12}^{2}}{s_{2 \beta} v^{2} t_{\beta}^{2}},
\end{aligned}
$$

$$
\begin{aligned}
\lambda_{3}= & \left(\frac{m_{H}^{2}}{v^{2}}-\frac{m_{h}^{2}}{v^{2}}\right)\left(c_{\beta-\alpha}-\frac{s_{\beta-\alpha}}{t_{\beta}}\right)\left(c_{\beta-\alpha}+s_{\beta-\alpha} t_{\beta}\right) \\
& +2 \frac{m_{A}^{2}}{v^{2}}-\frac{2 m_{12}^{2}}{s_{2 \beta} v^{2}} .
\end{aligned}
$$

Since we fix masses of all the physical states, the only free parameters are $\beta, \alpha, m_{12}^{2}$. The only constraint that is ever violated in the region of interest for our search is the region where $\cos (\beta-\alpha)$ is small and large $\tan \beta$. In this region, $\phi_{1}$ is decoupling, and $\phi_{2}$ becomes the SM Higgs doublet. Since our benchmark fixes the mass splittings of the additional Higgs bosons, it becomes strongly coupled in this limit. In this regime, the model is not under full theoretical control, although the boundary of this region is not sharply defined. We have indicated this region in our sensitivity in Fig. 1.

\section{APPENDIX B: $A \rightarrow Z h \rightarrow \ell^{+} \ell^{-} b \bar{b}$ SEARCH AT 14 TEV $300 \mathrm{fb}^{-1}$}

The most important backgrounds are $Z b \bar{b}$ and $t \bar{t}$. We use MADGRAPH5 to generate both signal and background and perform hadronization and the detector simulation with PYTHIA8 and DelPhes3. The $A \rightarrow Z h \rightarrow \ell^{+} \ell^{-} b \bar{b}$ benchmark cross section we used here is $50 \mathrm{fb}$.

We are interested in one OSSF lepton pair and two $b$-tagged jets. Our requirements are as follows:

(i) Two OSSF leptons with $p_{\mathrm{T}}>10 \mathrm{GeV},|\eta|<2.5$

(ii) Lepton isolation: $\Delta R<0.4, \quad p_{\mathrm{T} j} / p_{\mathrm{T} e}<0.15$, $p_{\mathrm{T} j} / p_{\mathrm{T} \mu}<0.12$

(iii) $b$-tagging: fake rate $=0.015$, efficiency $=0.75$ 


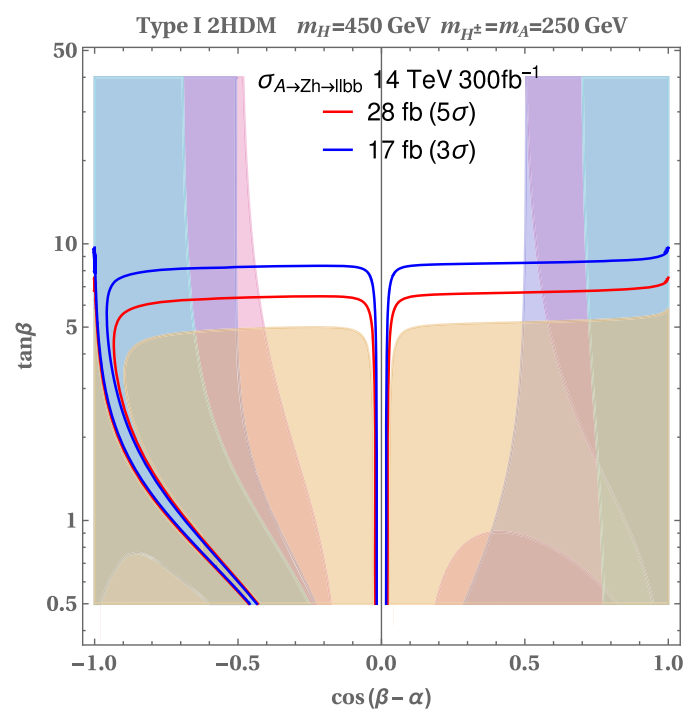

FIG. 12. $g g \rightarrow A \rightarrow Z h \rightarrow b \bar{b} \ell^{+} \ell^{-}$.

After preselection, we then optimize the cuts by maximizing $S / \sqrt{B}$. The final selections include

(i) $m_{b \bar{b}} \in[95,135] \mathrm{GeV}$

(ii) $p_{\mathrm{T} b \bar{b}}<85 \mathrm{GeV}$

(iii) $m_{\ell^{+} \ell^{-}} \in[81,101] \mathrm{GeV}$

(iv) $p_{\mathrm{T} \ell^{+} \ell^{-}}<85 \mathrm{GeV}$

(v) $E_{\mathrm{T}}<95 \mathrm{GeV}$

(vi) $m_{\ell^{+} \ell^{-} b \bar{b}} \in[220,280] \mathrm{GeV}$

We superimpose the cross section contours on top of the experimentally excluded regions from $8 \mathrm{TeV}$ run, see Fig. 12 .

\begin{tabular}{lccc}
\hline Event & $\sigma(\mathrm{pb})$ & Cut efficiency & Yield $\left(\mathcal{L}=300 \mathrm{fb}^{-1}\right)$ \\
\hline$Z b \bar{b}$ & 6.7 & $1.0 \%$ & $2.0 \times 10^{4}$ \\
$t \bar{t}$ & 23 & $0.04 \%$ & 2500 \\
Signal type I & 50 & $8.5 \%$ & 1300 \\
\hline
\end{tabular}

\section{APPENDIX C: $H \rightarrow Z Z \rightarrow \ell^{+} \ell^{-} \ell^{+} \ell^{-}$SEARCH AT 14 TEV $300 \mathrm{fb}^{-1}$}

The dominant background is ZZ. We use MADGRAPH5 to generate both signal and background and perform

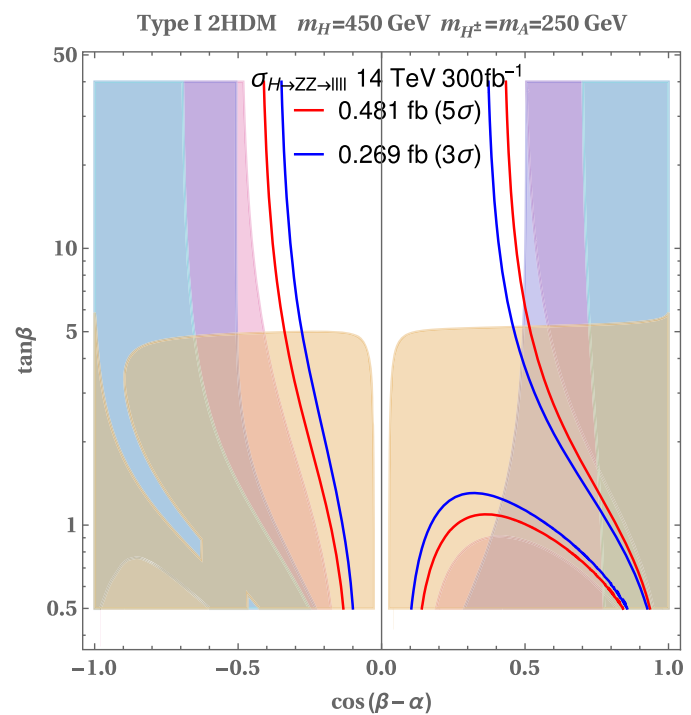

FIG. 13. $g g \rightarrow H \rightarrow Z Z \rightarrow \ell^{+} \ell^{-} \ell^{+} \ell^{-}$.

hadronization and the detector simulation with PYTHIA8 and DELPHES3. The $H \rightarrow Z Z \rightarrow \ell^{+} \ell^{-} \ell^{+} \ell^{-}$benchmark cross section is set to be $0.875 \mathrm{fb}$. We are interested in 2 pairs of OSSF leptons. We require

(i) Two OSSF lepton pairs with $p_{\mathrm{T}}>10 \mathrm{GeV}$, $|\eta|<2.5$

(ii) The three highest-pT leptons must satisfy $p_{\mathrm{T}}>20$, $15,10 \mathrm{GeV}$

(iii) Lepton isolation: $\Delta R<0.2, p_{\mathrm{T} j} / p_{\mathrm{T} \ell}<0.15$

After preselection, we then optimize the cuts by maximizing $S / \sqrt{B}$. The final selections include

(i) Two reconstructed Z's: $m_{\ell^{+} \ell^{-}} \in[55,105] \mathrm{GeV}$

(ii) $\Delta \phi$ between leptons in the same OSSF pair $<2$

(iii) $m_{\ell^{+} \ell^{-} \ell^{+} \ell^{-}} \in[365,475] \mathrm{GeV}$

We superimpose the cross section contours on top of the experimentally excluded regions from $8 \mathrm{TeV}$ run in Fig. 13.

\begin{tabular}{llcc}
\hline \multicolumn{1}{c}{ Event } & $\sigma(\mathrm{fb})$ & Cut efficiency & Yield $\left(\mathcal{L}=300 \mathrm{fb}^{-1}\right)$ \\
\hline$Z Z$ & 33.4 & $0.8 \%$ & 80 \\
Signal type I & 0.875 & $19.8 \%$ & 52 \\
\hline
\end{tabular}

[1] G. Aad et al., Observation of a new particle in the search for the Standard Model Higgs boson with the ATLAS detector at the LHC, Phys. Lett. B 716, 1 (2012).

[2] S. Chatrchyan et al., Observation of a new boson at a mass of $125 \mathrm{GeV}$ with the CMS experiment at the LHC, Phys. Lett. B 716, 30 (2012).
[3] ATLAS Collaboration, Measurements of the Higgs boson production and decay rates and coupling strengths using pp collision data at $\sqrt{s}=7$ and $8 \mathrm{TeV}$ in the ATLAS experiment, Eur. Phys. J. C 76, 6 (2016).

[4] CMS Collaboration, Precise determination of the mass of the Higgs boson and tests of compatibility of its couplings 
with the standard model predictions using proton collisions at 7 and 8 TeV, Eur. Phys. J. C 75, 212 (2015).

[5] D. Alves et al. (LHC New Physics Working Group Collaboration), Simplified models for LHC new physics searches, J. Phys. G 39, 105005 (2012).

[6] J. F. Gunion, H. E. Haber, G. L. Kane, and S. Dawson, The Higgs hunter's guide, Frontiers in Physics (Avalon Publishing, New York, 2000).

[7] J. Galloway, M. A. Luty, Y. Tsai, and Y. Zhao, Induced electroweak symmetry breaking and supersymmetric naturalness, Phys. Rev. D 89, 075003 (2014).

[8] S. Chang, J. Galloway, M. Luty, E. Salvioni, and Y. Tsai, Phenomenology of induced electroweak symmetry breaking, J. High Energy Phys. 03 (2015) 017.

[9] D. S. M. Alves, P. J. Fox, and N. Weiner, Supersymmetry with a sister Higgs boson, Phys. Rev. D 91, 055003 (2015).

[10] J. Alwall, R. Frederix, S. Frixione, V. Hirschi, F. Maltoni, O. Mattelaer, H.-S. Shao, T. Stelzer, P. Torrielli, and M. Zaro, The automated computation of tree-level and next-toleading order differential cross sections, and their matching to parton shower simulations, J. High Energy Phys. 07 (2014) 079.

[11] T. Sjöstrand, S. Mrenna, and P. Skands, A brief introduction to PYTHIA 8.1, Comput. Phys. Commun. 178, 852 (2008).

[12] J. de Favereau, C. Delaere, P. Demin, A. Giammanco, V. Lematre, A. Mertens, and M. Selvaggi, DELPHES 3, A modular framework for fast simulation of a generic collider experiment, J. High Energy Phys. 02 (2014) 057.

[13] CMS Collaboration, Identification of $b$-quark jets with the CMS experiment, J. Instrum. 8, P04013 (2013).
[14] CMS Collaboration, Performance of $b$ tagging at $\sqrt{s}=$ $8 \mathrm{TeV}$ in multijet, $t \bar{t}$ and boosted topology events, Report No. CMS PAS BTV-13-001.

[15] CMS Collaboration, Electron Reconstruction and Identification at $\sqrt{s}=7 \mathrm{TeV}$, Report No. CMS DP -2010/032.

[16] CMS Collaboration, Electron performance with $19.6 \mathrm{fb}^{-1}$ of data collected at $\sqrt{s}=8 \mathrm{TeV}$ with the CMS detector, Report No. CMS DP -2013/003.

[17] CMS Collaboration, Muon Identification performance: hadron mis-Id measurements and RPC Muon selections, Report No. CMS DP -2014/018.

[18] ATLAS Collaboration, Fiducial and differential cross sections of Higgs boson production measured in the four-lepton decay channel in pp collisions at $\sqrt{s}=8 \mathrm{TeV}$ with the ATLAS detector, Phys. Lett. B 738, 234 (2014).

[19] ATLAS Collaboration, Search for new light gauge bosons in Higgs boson decays to four-lepton final states in pp collisions at $\sqrt{s}=8 \mathrm{TeV}$ with the ATLAS detector at the LHC, Phys. Rev. D 92, 092001 (2015).

[20] CMS Collaboration, Performance of photon reconstruction and identification with the CMS detector in proton-proton collisions at $\sqrt{s}=8 \mathrm{TeV}$, J. Instrum. 10, P08010 (2015).

[21] ATLAS Collaboration, Report No. ATL-PHYS-PUB-2013014.

[22] H. E. Haber and D. O'Neil, Basis-independent methods for the two-Higgs-doublet model III: The CP-conserving limit, custodial symmetry, and the oblique parameters $\mathrm{S}, \mathrm{T}, \mathrm{U}$, Phys. Rev. D 83, 055017 (2011). 